\title{
Ueber die Zersetzung des Weingeistes durch Brom ;
}

\author{
ras \\ Dr. Carl Löwig.
}

Wie aus meiner Mronographie über das Mrom (das Mrom und seine chemischen Verbälthisse. Jieidelherg bei Winter 3829) hervorgelel, war ich sclon vor metrerna Juhren mil der Untersuchung über die Einwirkung des Broms auf Weingeist und $A$ ether beschäfligk. Ich habe in derselben angegeben, dass wenn Bron mi! wasserhalligem Weingeist zusammengebracht wird, starke Frhilzung einirill, die nach einiger Zeit so boch stcigt, dass leeliges Aufbrausen erfolgt durch kntwicklung bromwassersioffsauren Gases, und dass zugleich Bromwasserstoff gebildet wird, der durch Verdünnen mit Wasser oder dursh Kali abgeschieden werden karm. Thh habe gleichfalls angefïhrı, dass man dieselbe Verbindung erhält, weenn brom Jängere Zeil auf Aether cinwirkt und die bromhaltige Fliiszigkeit bis zu ein Drillel abdestillirt wird, wobei anfangs Bronwasserst offsäure, dann ein wasserklares Oel überdestillirte, welches ich damals firr Bromkolılenwasserstorf uich, während im Rückstand Bromkohlenstoff sirh befindet, welcher glcichfalls durch Wasser und Kali abgeschiedeu werden kann. Ferner ergieb1 sich ans meiner Nonographie, dass wenn Brom und Aether zusammengebra:lut wird, die Einwirkung so lhefig ist, dass bisweilen Entziindung hervorgebracnt wird, wobei sich der Aelher auf älnliche Weise wie der Weingeist zersetzt. Ich habe zugleich bemerkt, dass wenn man die älherische Bromlusung mit el was Wasser einige Zeit dem Sonnenlichte anssetat, die rothe Farbe nach und nach in ein Braun übergeht, und wenn man sodonn die Flürsigkeit mit Kalilösung schïittelt, augeablicklich der Geruch nach Essigälher hervortrilt, 
der sich durch Destillalion Jeicht rein sbscheiden lisst. Ich nabm damals an, dass durch Einwirkung des Broms aul Aether, Bromkohlenwasserstoff $\left(\mathrm{Br}^{4} \mathrm{C}^{4} \mathrm{H}^{3}\right)$ gebildet werde, ohne mish um die glcichzeitige Entstehung van Bromwasserstoffsäure zu kümmern, und dass dieser durch Wasserzersetzung unter Bildung von Bromwasserstoffsäure durch Sauersloffaufnahme in lissigather zerfalle.

Die Untersuchungen, die ich damals mil desn Brom vorgenommen, gehörten zu meinen ersten chemischen Arbeiten; es ermangelten mir nich! nur fast alle chemischen Geräthschaften, sondern aucle vorzüglich die chemische Umsicht, die man nur durch öfleres Lxperimentiren erlangt, und die damols angestellten Untcrsuchungen miissen daher al 3 sehr oberflichliche angesehen werden, ubgleich icis richtig beobachtet habe. Es war daher schon längst mein Vorhaben, diese Untersuchungen nochmals vorzunelimen, welches durch die wichtigen 'Arbeiten Liebig's uiber die Einwirkung des Chlors auf Alkohol doppelt wünschenswerth wurde. Ich habe dalier, da ich bercits im Besitze einer grossen Quantilät Brom bin, dieselbe wieder begonnen und werde in :iner lieihe von $\mathrm{Abhandlun-}$ gen meine Arbeiten bekarsnt machen. Ich beginne mit der Einawirkung des Broms anf obsolutem Weingeist.

Zur Zersetzung des Weingeistes durch Brom benutzle ich folgende A pparate: Eine zweilualsige hohe Flasche, in einer Sandkapelle befindlich, stand lufticht in Verbindung nit drei kleinen Flaschen, von denen die eine leer, die zweile mit elwas Wasser und die dritle mit Kalijauge gefüllt. war, und sämmitiche Flaschen waren miltelst einer Gasentwicklungsröhre mit einer mil Quecksilber gefiilten Glocke in Verbindung gesetzt. Die Flasche wurde $\%$ ein Drillel mit absolutem Wein; ${ }^{2}$,ist gefiillı, und das Brom durch eine hohe und gekrümmte Röhre, die bis auf den Boden der Flasche reichte, zugefiigl. Das Broln ward schnell und unler starker Wärme- 
enfricklung aufgenommen, die beim raschen Zugiessen des Brouns so zunimmt, Jass dis Miissigkeit in teblantes Kochen geruilh; je schneller das Zugiessen des Broms geschieht, desto hefliger ist die Einwirkung. Nach eitsiger Zeit hijrt das Kochen auf, und beim ferneren Zugiessen des Broms wird keine noue Eirwirkung mahr wahrgenortmen, ncch dann nicht, wemn die Flüssigkeit bis 2 mm Konhen erhilzt wird. Ich habe einen Theil der Flissigkeit bis anf ein Achlel Rückstand destillirt und yor Zeit zu Zeit Prom zugefügt, niemals aber konnte ich eine Reaction des Hroms wahrnehmon. 6 Unzen Weingeist erfordern bis zum Auflüren der genannleu Erscheinungen 15 - 16 Ifnzen Brom.

Wiklurend der Linwirkung des Broms findel slarke Gasentwicklung 3latt, in dur leeren Flasche sammelt wich eine be deutende Menge ciner sehr flücbligen, rüthlioh gefärblen, slark und angenehm riechenden Flüssigkeit, iber welche eine farblose Flüssigkeit sich befindet. Das Wasser in der andecn Flasche wurde stark sauer und unier derselben ist gleichfalls cine farblose Miussigkeit von demselhen Geruche wie die röthlicte in der leeren, und das Kali in der aritten wer zum Theil gesättigt; in der Glscke wurde eine nichl bedeutende Menge Gas erhalien.

Nachàcm die Finwirkung des Brome nachgelassen, wurde die Flüssigkeit in der Flasche noch mchrere Stunden im Kochen erhalien, und von Zeit zu Zeit neue Antueile Brom zugefigt. Die Temperatur, nei welcher dic Flïssigkeit siedet, ist anfange $45-50^{\circ}$, sie axeigt aber wech und wach bis auf $100^{\circ}$. Wibrend des finchens sanmelle sich noch eine bedeutende Menge oben gentinter Substanzen an. Nachdem die Destillaion. eine Zeitlang gedauert, setzen sich an den Wundungen und dem Boden der Flasche Flocken ab, die aber bei Corlgesetztem Erhitcen wieder verschwinden und wichl mehr rum Voraciein kommen, deshnh auch niclul untersucht wer- 
den konnten. Siachdem nur noch ein Bechsiel der ganxen Masse in der Deatillationsflasche vorhanden war, wurke dio Destil. lation unterbzochen. Die noch suriickgebliebene Flüssigkgit zeigte folgende Figenschafien: sie hallo eine gelbruthe Farbe, cinen eigenthimlichen durchdringenden, stark zu 'Thränen reizeaten Gexuch und einen hüchst scharfen zusammenziehenden uud zugleich suren Geschmack. Ihre Consistenz ist ölig; sie fühlt sich feltig an und giebt anf Papier Fetlffecken. Sic ist schwerer als Wasser und schwimmt uater derrselben in Gestailt eines dicklichen röthlichen Breis; wird sie längerer Zeit mit viel Wasser geschüttelt, zo lüst sio sich rollständig darin auf. Mit Kaliauflïsung zusammengebracht verliert sie augenblicklich dio röthliche Farbe; mun erhält Bromkaliun nebst etyras bromsauren Kali (ein Beweis, dass die Farbe von iberschüissigem Brom herrührt), und nach einiger Zeit scheidet sich, nachdem die Kalibösung roß dem raspectiven Oele getrennt wird, aus der Katilösung eine angenebm riechnnde ölige Fliissigkeit ab, welche sich auf dem Boilen des Gefässes ansarnmelt. Mit Alkohol und Acther ist sie in allen Verbälinissen misclibar. Mit der Luft in Berührung gebracht, bilden eich in dersellonn weisse Krystallen.

\section{Unterwuchung der überdestillirten Flüsigkeiten.}

Wie bemerkt wisde, ammellen sich in der lecren Flo. sche zwei verschiedene Jlüssigheitan $a n$, von denen die eine schwer und röblich gelb gefärtst, die andere hingegen ohne Farbe ist. Die röthliche Farbo der schweren Flüssigkeit rülarte ron etwas Brom her, welches ich auf keine Weise als durch Kali oder durch anhaltendes Waschen mit Wasser entfernen konnte. Die entfärble anfangs wassecklare Flïsoigkeit trïbte sich mach einigen Tagen, und es setzle sich an den Wandungen des Gefässes eín weisser fester Kürper ab. Nach 8- so Tagen war sie farbius und vollkommen durchsichlig. 
Sie wurde bei der gelindesten Wärme destillirt, wobei noch eine geringe Menge der waissen Substalız zurückblieb. Die destillirte Fliissigkeit verhält sich wie folgt. Sie het einen starken angenchm siisslichen, hinlennach etwas brennenden lang anhallenden Geschmack und cinen ätherartigen durchdringenden Geruch. Bie cutzündet sich nur schwierig, und brennt, wenn man sie miltelst eines Glasstabes in die Spirituslempe bringt, mit einer sehr schönen grinen Flamme ohue Russ und mit starkem Geruch nach Bromwaseerstoffsäure. Durch ein glïhendes Glasrohr geleitet zerfillt sie in ölbildendes Gas und Bromwasserstoffsäure; gliiht die föhre weiss, 80 selzt sich in derselben Koble ab. Von Wasser wird die Flïssigkeit nur wenig a'sfgenommen, retheill ober doch demselben den sïsslichen Geschmeck und ätherarligen Geruch, Aether und Alkshol mischen sich mit ilır nach allen Verhälinissen. Von Salpetcrsäure und Schwefelsäure wird sie nichi zerstört, anch nicht von Kalium. Uhr spec. Gewicht ist 1,40 . Alle diese Eigenschaften zeigen, dass diese Flüssigkeit die von Serullas dargeslellte Bromwasserstoffnaphta ist; da Serullas diese Substanz. nur sehr unvollkommen beschrieben hat, so glaubte ich, die Eigenschalten dieser Naphta etwas ausfihrlicher angeben zu müsscn.

Die erste Sulstanz, die sich aus der Bromwassersioffnaphita ausgeschieden hat, ist weiss, undurchsichtig, fihlt sich fettig an und ist schwerer wlo Wasser. Sie schmilzt bei gelinder Wärme und verdampft unveräudert. Der Geruch ist äusserst gewürzhaft. Dex Geschmack scharf und brennend, erwirmend, hintennacb kihlend und angenehm süss. Wird die Substanz in die Weingeistflamme gebracht, so verbrennt sie mil grünlicher Flamme und unter Ausstossung bromwassersloffaaurer Dämpfe; aus der Flamme genonmen, verlöscht sie sogleich wieder. Leitet man dic Dämple über glühendes Bisen oder Kupfer, 80 erhäl man Bromeisen oder Bromkupfer 
und Kohle ohne Spur eines brennbaren Gases, worams hervorgelit, dass diege Substanz fester Bromkoblenstoff ist. Leider hatte ich zn wenig zur Anstellung einer quanitiativen Analyse. In Alkohol und Aether ist er lïslich und die Aufösungen werden durch salpetersaures Silberoxyd nicht gefallt. Ohne Zweifel ist dieser Bromkohienstoff derselbe, den ich früher dargestellt und in meiner Monographie beectirieben babe; ich komme übrigens bei einer andern Gelegenheit nochmals auf denselben zurürk.

Dic andere gleichzeitig mit der Bromwasserstoffnaphla in der leeren Flasche erbaltene farblose Flissigkeit besitzt einen scliarfen Gesrhmack, der zugleich situer ist und einen äusserst durchdringenden, Jie Augen zu Thränen reizenden Geruch, der besonders beim Erwärmen der llïssigkeit hervortritt. Ein Theil theser Flïsigkeit wurte mit Aether geschüttelt, und er erhielt dadurch ganz den Geschmack und Geruch der damit belaandelten Flissigkeil. Lüsst man den Aether freiwillig verdunsten, so bleiben zulet:al kleine weisse Krystalle zurück, auf die ich weiter unten ausfülurlich zu sprechen komme. Vorliufig bemerke ich nur, dass diese Krystalle Yerbindungen sind einer neuen flïssigen Substanz mit Wasser, weiche in der Zussmmensetzung und sonstigen Eigenschaften, lis aui wenige Ausnahmen, ganz der Verbindung gleicht, die Liebig durh Zerselzung des Weingeistes mittelst Chlor erhielt, und Chloral genamit hat, weshalb ich diesen Körper Bromal nenne. Die lilissigkeit, welche mit Aether behandelt war, besitzt einen sauer und zugleich scharfen Geschmack, übrigens war der Geschmack lange nicht mehr so scharl als vor der Pehandlung mit Aether; ganz aber konnte die scharfe Substanz durch Aether nicht entzogen werden. Wird die mit Aelter behandelte Flüssigkeit mit einer Auflösung von reinem Natron gesättigt und sodann destillirl, so gelut ein farbluses wässriges Destillat über von äusserst 
sussen Ceschmack und angenehmen durchdringenden Gernch, und auf dem Boden der Vorlage sammein sich einige Tropfen einet wasserklaren Fliiszigkeit an von tüssigem Bromkohlenstoff, derselbe ist jedoch nicht sehon in der Flïssigkeit enthalten, sondern wird exst durch Binwiskung lies Nutrons cenf das noch in derselben aufgelöste und durch Aether nicht vollsthindig catcogene Brumal gebiliet. Destillirt man; nachdem das Waseer geschmacklon überulestutirt, den Kücksiand in der hetorte mit einer ganz kleinen Quantitüt Selıwefelsäure, so erhiilt man cine saure Hüssigkest obne Spur von Bromwassertfulfsüure. Wird diese saure Flissigkcit mit rothem Quecksilberoxyd exhitat, so wird dikses sogleich unter Anfbrausen zu molallisehem Quecksilber reducirt. Da ich diese Bäure bei einer andern Geiegenbeit, wic ich weiter unten zeigen werde, in grïsaerer Menge erhielt, so hake ich damit nahere Unier. suchungeu vargenorninen, und mich, besonders durch Umtersurhung melirerex danit dargestellien Salze mittelst Schwefelsäure, volłkommen überzeugt, dass die fragliche Sänre keine andere als Ameiscnsäure seyn kann. Otme mich in die Btreiligkeiten l.icibig'o und Göbel's zu mischen, kann ich doch nicht unerwikn! lassen, dass ich das Verbalten der Ameisensäure zu Quecksilberoxyd, wenn dieselbe frei von Bromwas. serstofiäure ist, genau so fand, wie Liebig angegeben hat. Auch die Ameisensäure ist ein Zersetzungsprodukt dies Hrom. als. Ist die Amciseusäure abdestillirt, so bleibt in der Rotorte eine Aufiösung von Bronnatriun xurick; bromsaures Natron befindet sirh nicht im Aückstande,

Da" Wasaer in dez a weiten Flasche wax stark sauer und zegte last gar kitucr scharfen Geschmack und Gersach. Wird die Flüssigkeit mit Natron gesättigt und deskliht, so erhält man keinen Bromkolicustoff, eben so erbiti man durih Desillatinn tait weng Schweftsäne keine Bromsisure, uml sie ist duber blos als eine wässrige Lüsung you Bromwagserstoff- 
säure anzusehen. Das Kali in der drillen. Flasche ist zum Theil mil Bromwassersıfofsäure gesälligt, um! die über Quecksilber aufgefangene Luft bestcht nach einem unil derselben vor. genommenen eudiometrischen Versuche aus atmosphïrischer Luft ohne Spur von Kohleneiure und ïbersebäasigem Saverst off.

Hieraus geht also hervor, dass die Subslanzen, die sich bei. der Finwirkung des Broms auf Weingeist gasförmig entwickeln, folgende sind: Bromwasserst offnaphta, Bromwascrstoffsïure, fester Bromkohienst off, Wasser und Bromal, welckes durch Einwirkung von Al. kali sich in Ameisensïurc und flïssigen Bromkohleustoff unter Bildung von Brommetall zersetat.

Obgleich die Menge des Bromals, weiches in ner ersien Elasche exhalten wurde, nur sehr wenig betrögt, und das meiste in der Destillutionsflasche zuriickbleibt, daher auch bei dem beschriebenen Versuche nur eine kJeine Menge flüssiger Bromkohlensioff erbaiten werden honnte, so will ich Joch, bevor ich in der Beschreilung meiner Versuche weiler schreite, die Eigenschatten und Zusammensetzung dieser Substans miltheilen, die ich wir auf andere Weiee in grösserer Menge verschaff habe.

\section{Flüssiger Bromkchlenstoff.}

Diese Vertaindung erhäit man ausser durch Zersetzung des Bromals noch durch Zersetaung verschiedener underer organischer Verbindungen; ja ich habe Ureache zu glauben, dass sie sich fast aus allen solchen organischen Körpern, die wir als indifferent bezeichnen, darstellen lässt, wenn man dieselben in verdünnten Zustande mit Brom behandelt; denn bei allen Destallationen diezer Kürper mit Wasser und Brom trhălt man ein Destillat, weichen ganz die Figenschaften dieses Brum. kuhlenstoffs zeigt; grössientheils aber bildet er sich in so gerin- 
gor Menge, dass or in dem überdestillirten Wasser aufgelöst bleibt.

Den Bromkolslenstoff, von dem hier die Rede ist, habe ich durch Zerseltung des Bromals miltelst Kali oder Natron erhalten. Nan erhält ihn ganz leicht rein, indem man das Bromal mit einer Aufüsung des Kalis destillirt. Wendet man zil wenig Kali an, so deslillirt auch noch Bromal mit über. Die erhallene wasserklare Fliissigkeit bebandell man, narhitem man sic rom überstehenden Wasser grüsstentheils befreit! hat, mit Schwefelsätsre zur Entfernung sämmllichen Wassers, und erbäll sodann den Bromkohlenstoff rollkommen rein.

Dicser Bromkohlenstoff ist eine wasecrhelle, tropibare Flïsigkeit von 2,13 spec. Gewichte und äuseerst angenehmon und gewürzhaften Geruche und süssem eigenthïmulichen $G_{t-}$ schmick, welchen er dem Wasser, in dem cr uur sehr wenig libslich ist, mittheilt. Er Jässt sich nicht entzünden, und wenn man einen damit befeuchleten Glasstab in die Weingeistfamme bält, bemerkt man eine trübe russigte Flamme, die nach eini. ger Zeit enlïscht. Kalium wirkt in dec Kälte auf diesen Bromkohlenstoff nichl ein, aber in Dampfe desselben crhitzt, verbrennt er mit lebhaftem Lichte, unter Absalz von Kohle, zu Bromkulium. Wird der Bromkohlenstoff in Gasform ïber glühenden Faryt und Kalk grleitet, 60 erhält man Brominetalle und kohlensaures Metalloxyd neben etwas Koule. Werden die Dimpfe iber glühendes metallisches Fisen oder Baryl geleitet, su erhält man, indem sich der Bromkohlenstoff vollkammen zerlegt, Brommetalle, die mit Kohle umgeben sind, aher sonst keine bremnbar gasförmige Substanz. Wird er durch eine gliihende Glasröhre geleitet, so zerfallt er zuma Theil in Kohle und Bromgas, ein Theil aber geht unveraindert durch die Röhre. Er ist in Weingeivt, Aether und flüchtigen Oelen löslich, und er selbst ist ein Aufböeungomittel für Jod, 
Schwefel undPhosphor, welche beide letzteren Substanzen jedoch nur in geringer Menge aufgenommen werden.

1,53. Grm. Bromkohlenstoff, welche in ein kleincs Glaskügelchen gewogen, wurden auf den Boden einer un einem Ende zugeschmolyeneis Glasröhre zebracht, die Föhre mit reinem gebrannten Kalk angefiillt, dieser sodann nach und nach in schwaches Gliken getracitis, und der Bromkohlenstoff, uuter Berücksichligung der von Liebig bei des Analyze des Chlorkuhlenstoffs angegebouen Vorsichtomanssiegein, über denselberz geleitet. Nach geschehener Lersetzums wurde aller Kalk in Salpetersäure aufgelöst, die Aufösung von der Kohie abfiltrirt und mit salpetersaurem Silberoxyd gefäll. Es warden 3,316 Gra. Bromsilber erhalten, welche 1,378 Grm, oder 93,6 p. C. Brom enteprechen. Ein anderer Versuch gah, $92,8,9$ p. C. Brom. Nimmt man das Mittel beider Versuche, so erhält man 92.99 p.C. Brom. Wird nun das Fithlende für Kolhlenstoff angenummen, so bestehen 100 Theile flüssiger Bromkublensloff aus

$$
\begin{aligned}
& 92,99 \text { Brom, } \\
& 7,01 \text { Kohlesstoff. } \\
& \hline 100,00 \text { flössiger Bromkohlemstoff. }
\end{aligned}
$$

\begin{tabular}{|c|c|c|c|c|c|c|}
\hline \multirow[b]{2}{*}{1 Alom } & \multirow{2}{*}{\multicolumn{2}{|c|}{ Kohlenstoff }} & \multicolumn{3}{|r|}{ Berechnet. } & \multirow{2}{*}{$\begin{array}{c}\text { Gefunden. } \\
7,01\end{array}$} \\
\hline & & & & 6 & 7,11 & \\
\hline $1-$ & Brom & • & • & 78,39 & 92,89 & 92,99 \\
\hline 1 Atom & Brom & & & 84,99 & 100,00 & 100,00 \\
\hline
\end{tabular}

Dieses Verhältniss spricht am nächsten fïr gleiche Atome Brom und Kullenstoff.

Diese Zusammensetrung stimm mil der Liebig'schen Analyse des, durch Zersetzung des Chlorals mitteist Alkalien erhalienen Chlorkohlenstoffs, welcher derselben aus 4 Atomen Kohlensioff und 5 Atomen Chlor zusammengeselzt fand, nicht überein. Ich gestehe, ich schätze Liebig als analytischen Chemiker so koch, dass ich selbst gewiss bin, meine UnterAnnal. d. Pharm. IIL Bds. s IIft. 
suchungen für unrichlig zu hallen, indem das Bromal bei seiner Leroetzung so sehr mit dem (hloral ibereinstimmt; beachtungswerth hleibr es übrigens doch, dass mein erster Versuch so gand mit der Berechnung ühreinkommt. Sollte vielleicht der I. i ebig'sche Chlorkuhlenstulf ein Gemenge seyn von Andertbalb Chloriohlenstoff nit Einfach Chlorkoblensturfe? Merhwïrdig ist es imuner, dass wemn der fragliche Chlorkolulensloff durch eine g] ühende Glasröhre gedeit el wird, lerselbe in Anderthalb Cllorkohlenstoff zerfallt, da docb, wie aus F a r a d a y's Unlersuchung hervorgeht, der Anderthalb Chlorkohlenstoti, aof aihniche Wcise behandell, Einfach Chlorkohlenstoff liefert *).

*) Nach dem richtigen Atomeugewicht des Xohlenstnffs berechnet, beateht der einfache Bromkohleustoff in 100 Theifen aus

99,27 Brom,

7,73 Kohlenstoff.

Ein Bromikohlenstoff, analog in seiner Zusammensetzung dem Chlorkohlenstoff, welcher bei Zersetzung das Chlorals mit wïusrigen Alkalien erzeugt wird, würde in 100 Theilen enthalten

$$
\begin{aligned}
& \text { Yi,04 Brom, } \\
& \text { 5,86 Kohle. }
\end{aligned}
$$

Mit der letzterea Zusammensetzung stimmt der Brongehalt, so wie thn die erste Analyse Lö w i g's gegeben hat (\$3,6p. C.), bis auf 0,46 p. C. Rrom genau uberein. In allen Fällen ist das Mittel der beiden Bestimmungen wicht $9 \pm, 99$, sondern 93,24 p. C. Brom. Diese Quantitat ist gröcser als das theoretische Resultat vorauscotat, chenn damach bat L ö wig statt eines wahrschcinlichen Verlusies in der ersten Aralese 3 p. C. Bromsiber z: viel erhalten, was genaner berechnet auf 1,532 Theile Bromkohlenstoff 4,85 Theile Bromsiber ausmacht. Nach der andern ist der Ueberschuss 1,5 p. C. BramsilberDies halte ich nun nicht fuir wahrcheinlich, in Gegentleile scheinen mir diese Analyoen uber die analoge Zusammen- 
Untersuchung der in der Dastillationsfasche surü̈chgeblicbenen Flüssigkteit.

Nach einigen Tagen hatte sich der Rückstand in der Flasclie in 2 wei Schichten getrennt, die von einander geschieden wurden. Beide so erhaltene Flüssigkeiten baiten eine schwach gelbliche Farbe, ölartige Consistenz, gaben auf Papier Fettflecken, und waren ausgezeichnet durch einen durebdringenden, höchst beissendea Geschmack, und einen anfangs angenehmen, dann aber жcharfen und stark zu Thränen reizenden, fast unerträglichen Geruch. Lackmurpapier zeigte in beiden dic Anwesenbeit von freier Säure. Mit wenig Wasser vermischi scheidet sich aus beiden ein gelblicher Körper, und das dartiber stehende Wasser mahm ganz denGeruch und den Geschrnack der Flissigkeit selbet an. Wurde viel Wasser hinzugesetzt, so erfolgte vollständige Lösung. Wirden beide Flüssigkeiten eine Zveitlang in einer offenen Schale der Luft ausgeetzt, so schied sich nach einiger Zeit eine gelbliche krystallisirle Bubstanz ab, während ein klelner Theil flissig blieb, der abgenummen keine Krystalle lieferte, nber nach einiger Zeit vollatändtg ver-

setzung dieses firemkohlenstoffe mit dem Chlorkohlenst off $\mathrm{Cl}^{5} \mathrm{C}^{\text {s }}$ keinen Zneifel zu lassen.

Gegen die oben ausgesprochene Meinung, dass der Chlorkohlenrtoff $\mathrm{Cl}^{5} \mathrm{C}^{2}$ ) ein (iemenge seyn kanan ron $\mathrm{Cl}^{2} \mathrm{C}$ und $\mathrm{Cl}^{*} \mathrm{C}$, opricht der Umstand, dass dex Einfach Chlorkohlenstoff bei $71-77^{\circ}$ siedet, withrend der Siedepunkt des Cls $0^{\circ}$ 60,80 betrügt. Auch werden Dämpte von festen Chlorkohlenstoff durch eine glühende Glasröhre getrieben, der man, weil sie weich wird, kciae so hohe I'emperatur geben kann als einex Porzelianröhre, nicht oder kaum zersetzt; dit Entstehung des Anderthalb Chlorkohlenstoffs aus $\mathrm{Cl}^{\text {s }} \mathrm{C}^{*}$ erklïrt sich genügetid dureh den Absatz von Koble.

J. L. 
dunstete. Lus diesen Versuchen ging daher horvor, das beide Flïsigksiten verschieden, aber walirscheinlich diegelben 8 ubslonzen esthielten, iaden jedc einzein die schon oben angegebenen Verhältnisse der grmenglen Substanx zeigte.

\section{Lhterswchung der untersten Schicht.}

Ein'Theil dex unitisien Srhicht wurde cinigemal mit wenig Wasser geschüttelt und das Waster von dem zu Buden gefallenen üligen Kürper getrennt. Das Wasser hatte einen stark sauren (jeschmack, einen töchst durchdringenden eigenthümlichen, afark zu Thränen reizenden Gerucb und eine gelbliche Farhe. Ein Theil diesar wässrigen Lösung wurde 80 lange mil Aether behandelt, bis das Wasser fast allen Geruch und Geechmack verloren hatte; gänzliche Entriehung des Geruchs nnd Geschmacks waraber nicht möglich, und es blieb folglich ein ïbrigens kleiner Theil von der im Wasser aufgelösten Subsianz zuriick. Von der gelbgefärbten ätherischen Lösung wurden zwei Drittel bei ganz gelinder Wärme abdestillirt. Der überdestillirte Aelber hatte einen schwach scharfen Geschmack und Geruch und der Rückstand in der Retorle zeigte dieselben Eigenschatten, wie die mit Wasser behandelle Flüssigkeit.

Die wässrige Flüssigkeil, welcher durch $\Lambda$ ether grösstentheils die scharfen Eigenschaften entzogen waren, wurde suil Natron gesälligt und die gesälligte Fliissigkeit in einer Retorte destillirt, wobei ein Destillat erhalten wurile von angenebm älherischem Geruch und süsolichem Boschmack, nebst einigen Tropfen von flissigem Bromkohienstoff. Als nur noch reines Wasser ïbewdestillirte, wrode das in der Returte Zurückgebliebeut in einer offenen Schale bei ganz gelinder Wärne zur Trockne cingedampft. Der Rückstand halle eine brïunliche Farbe und einen rein salzigen Geschmack.

Ein Theil des salzigen fückstandes wurde in wenig Wasser aufgelöst und mit verdünnler Schwefelsäure, die aber nur 
in kleiner Menge zugefügt wurde, destillit. Dit Vorlage stand durch eime Schenkelröhre mil Kalkwaeser in Verbindung. Fis ging ein schwach saures Destiltat über, welches von salpelersaurem Silberoxyd fast gar nicht get ribl wumde; Ent wicklong von Kohlensäure fand während der Destillation nichl stali; es erfolgte wenigblens niclut die geringste Trübung des Kalkwassers. Nachidem nux noch reines Wasser iiberdestillirfe, wurde eine neue Portion Schwrefeleäure zum Rückstand in der Retorte gesetzt, und nachdem das frühere Destillat entfernt, ron neuem destilirt, wobei eine stark saure, Bromwasserstoffsäure exthaltende Flüssigkeit erhalten wurde. Beide Destillate wurden mit Baryt geaälligt und zur Krystallisation eingedampft. Das erste Destillat lieferte Krystajte, die in allen Verhältnissen mit amseisensaurem Baryt ühereinkamen, und das 2 weite Brombaryum, welches noch mit etwab ameisensanrem Baryt vermischt war. Um mich zu üherzeugen, ob die Ameisensäure blos durch Zersetzung des in Wasser noch aufgelösten Bromal ertstenden, oder ob sie schon vorbanden war, wurde ein anderee Theil der wässrigen Flïssigkeit, welcher zuvor thit Acther behandelt war, einer gelinden Destillation unterworfen nud mit der Vorlage anehre Flasehen luftdicht in Verbindung geselzt, die Destillation abee bald unterbrochen. Das Desilltut halts ganz den eigenthümlichen Geschmack des Wasters und reagirte deullich sauer auf Lackmns. Salpetersaures Sulberoxyd brachle im Destillat eine geringe weisse Trübung hervor. Das Destillat wurde hierauf fast bis zal gïnzlicher Entziehung des scharfen Geschmacks wil Aether geschütlelt und sodam der, in der wässrigen Flüssigkeit noch zurückgehallene, Aether an der Luft verdunsten gelasken. Beiru Kuchen cines Theils der wässrigen cauren, yom Aether befreiten, Flü̈. sigkeit mit Quecksilberoxyd enlwickelte aich sogleich Kohlensäuregas, unter Reduktion des Quecksilberoxy ds. Ein anderer Theil wurde kait Baryl gesialigl, und nach den Abdampien 
ameisensiurer Baryl erhalten. Wird der Aether, welcher die scharfu Substanz aus dem Wasser aufgenommen batte, geliade destillirt und der scharf schmeckende Rückstand mit Barylhydral xusammengebracht und aberuals destillirt, so geht flïssiger Bromkoblenstoff iber und als Rückstand bleibt emeisensaurcr Baryt und Brombary:um. Hicraus gebt also hervor, dass in der wïssrigen Flïssigkeit schon Anteisensäure und Bromwasserstuff fïure enlhalten waren, dass aber auch zugleich durch Einwirkung von wässrigen Alkalien Amejsensäure, Bromwaserstofisäure und Bromkohlensto if gebildet werden, und folglich die scharfen Eigenschaften der wässrigen Flüssigkeit von aufgelöstem Bromol herriluren.

Um mich nun noch zu überzeugen, ob boi der Zersetzung des Weingeistes durch Brom nicht auch zugleich Bromsäure gebildet werde, wurde ein bedeutender Theil der wässrigen Lösung mit salpetersaurem Silberoxyd gefallt, aber keine Spur yon bromsaurem Silberoxyd erhalten.

Oben wurde bemerkt, dass wenn die untersle Schicht mit Wasser, dieses alsdann mit Aether behandell, und dar Aether abdestillirt wird, eine Flïssigkeit zurückbleibt, die im Wesentlichen die Eigenschaften der mit $\mathbf{W}$ asser behandelten Flïssigk rit zeigt. Unn sich diezen Körper in grösserer Menge and frei von Ameisensäure und Bromwasserstoffëure zu verschaffen, wurde ein anderer Theil der untersten Schicht sogleich mit Aether behandelt. Nach einigen Stunden bildeten sich zwei Schichten, die unterste besland ans wässriger Ameiseusäure und Bromwasserstuffsäure und die ohere war Aether, welcher die ibrigen liestandtheile aufgenommen hatte. (Hierbei muss ich jedoch bemerken, dass wenn man die Flüssigkeit mit Aethet schuittell, eini: grosse Menge Aether angewandt werden muss, irdem sich in: crtgegengesetzten Falle der Aether mit den ausser A meisensäure und Bromwazserstoffsäure vorhandenen Substanzeu zu einer zu Boden lallenden Flüssigkeit vereinigt). 
Um nun diese Subslanzen frei von Aether zu erhaltes, wurde dersolbe bei der gelindesten Wärme abdestillirt. Der erhahtene Kückstand ist ein Gemenge von zwei bestimmt von einander verschiedenen Körpern, die sich aber nur äusserst schwierig rein darstellen lasken. Diese Schwierigkeilen werden noch vergrüssert wegen derLeichtigkeit, mit der sie mit $\Lambda$ ether, Weingeist, Hydrobromsäure und selhst Wasser verbindbar sind. Dic Subsionzen in Aether aufzulösen und den Aelber abzudestilliren, hat aneser dem Nachtheil, die Körper nichi rein, sondern mit Aether vermischt zu erhalien, such den, dass cin bedeutender Theil mit dem Aether iblerdestillirt, aus dem gie nicht mehr zu erhalten sind. Die grösste Schwierigkeit besteht aber in der Trennung der verschiedenen Körper.

Die von der Destillation des Aethers zurïckgebliebene Substanz besteht, wie ich mich auf das Bestimmteste überzeugte, ans Bromal und aus schwerem Bromäther. Von dem schweren Bromäther wird in dieser Abhandlung nicht mehr die Rede seyn, weil ich die Eigenschaften desselben bei einer andem Gelegenheit in einen der folgenden Hefte der Annalen erwähuen worde.

Bringt man des Gemenge von Bromal und schwrerem Bromalher mit concentrivter Schwefelsäure zusammen, so wird eine bedeutende Wärmcentwicklung wahrgenommen, unter Entbindung einer grossen Menge bromwasserstoffsaurer Dämpfe. Schüttelt man das Gewenge von Bromal und schwerem Bromather längere Zsit mit Schwefelsäure, so sondert sich nach einiger Zeit auf dem Boden des Gefässes eine wasserklare Flïssigkeit $a b$, und die darüber stehende Schwefelsäure hesitzt slarke rauchende Ejgenschaften, die von Bromwaseertoffsänre herrülıren. Da reines Bromal durch Schwcfelbäure nichl zersetzt wird, so glaubte ich anfongs aul diese Weise den schwe. sen Bromäther zerstören und so das Bromal rein erhalteu zu können. Ich destillirte dalter die von der Schwefelsäure be- 
fruile Cliizsigkeil ibter zuvor gelöschen und wieder stark aus. geffranmen Kalk, und arhielt eine wasserklare Fliassigkeit, die von des Sechwefeigäure nichi wieder cersetzt wurde. Do die Wörmeentwinlung: areicise hein Zusanmenmischen der Sniwgteliaure mit dern Gemonge von Brumal und schwerem

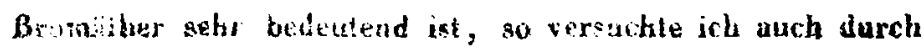
Qnatillation dieskg Geinenges unil Schwefelsäure das Bromal sugleisi rein ze erbaliten. Ich vermischte es daher mil der ti Beachen Menge Solwefolsaure and unterwarf das Ganze sines Destillation, Dic Schweliskäure fing bald an sich zu schwäsen, es ellwickelte sich eine Menge bromwasserstotisaurevi Gases nebst schwefliger Säure taid Kohlensäure, und zutblein destillirte eine farblose Flïssigkeit über, weiche durch gelintles Erwärmen vollkomnen von der schiwefigen Süure he. fwit wurde. Aker schon der rezändeste Geruch, den sorohl die mit Schwefelsäure beliendelte und über Kalk reclifivirle, su wis auch die mit Scilwefelsäure destillirte Fitissigkeit zeigle, war ein Beweis, dass auf diese Weise das Bromal nicbt rein erlialten werden konnte, und wahrscheinlich wor es anii Bromk ublenstoff, der sich bei der Zersetzung des schweren Mromäthers schon gebilde1, verunreirigl, für welche Meinung besonders der sitssliche Gernch Veranlassung giebi.

lch habo beatite erwälat, dass wenn der Riickstand in Her Destilationsflabche der Luft in Hachen Schalen ausgesetzt wixd, ein Thein devon fest wird, während ein anderer Theil tisssig bleib, der bei fornerem A ussetzea an der Luft nicht in des festen Zustend äbergeht und nach einiger Zcit vollständig sich verllücbigt. Der Theil, welcher fest wird, ist bromal, unbi der andere schwerer Bromälher.

ia dic Budtung der kirystalle in der Ferbindung des Waspesti der Luft mil demBromal herubs, so figte ich zu dem fierienge von Btomat und schwerom Bromäther eine kleine Menb. Wiasser, weiches sich gieicherformig damil vermischle, und 
liess dus Ganze in eincon verschlossenen Gellsse steben. Vebrigens hatte aich nach to Tagen nicht das Geringste abgeschieden, und es scheinl daher die Billung der Krystalle mil der Verdunstung des Bromälhers im Zusaınmenlange zu stelen. Ich brachle daher cine grusse Meuge Brumal mit schverem Bromälher gemischt in eine flache Schale unit der Luft in Berillrung, und sach wenigen Stunden thalte sich eine Menge der genarnten Krystalien gebildet. Dieselben besitzen eine gelbliche Faxbe und können von dem noch arhängenden schweren Bromäther durch Urücken zwischen Löschpapier gröestentheils befreit werden. Cianz rein erhielt man sie aber nicht, demn miecht man si! nit Schwefelsäure, so findet noch Pil. dung bromwasserstoffaturer Dïmpfe stalt. Viel reiner erbält man die Krystalle, wenn man das Gemenge von Bronsal mit schwerem Bromälber zuerst mit Schwefelsäure deslillirt und das ïbergegangene Destillal der feuchten Luft aussetzt. Werden nun diese Krystalle mit der sechsfachen Menge Schweílsä́ure verwischt und mil derselben gelinde erwärmt, so scheidef sich unter derselben eine wassurklare Flïssigkeit ab, die abgenommen und zur Frtfernung sïmmtlicher Säure, über zuvor gelöschten und dann wieder ausgeglïhten Kalk recificirt, das reine Bromal darstellt.

\section{Bromal.}

Das anf dic angegelsene Art dargestellte Bromal zeigt folgende Eigenscbaften. Es ist cine wasserhelle Flüssigkeit, die gich etwas fellig anfiibl! und auf Papier. Fetllecken macht, die aher nack eirniger Zeit wieder verschwinden. Sein spec. Gewicht ist 3,34 und sein Siedepunkt liegt übex $100^{\circ}$. Es lässt sich olne Veränderung destilliren; schmerhl bơnhst scharf und brennewd und lange anhaltend, und sein Gernch ist eigenthüm. lich durchdringend und die Angen heflig zu Thränen reizend. Im Wasser ist es leich! löslich; auch lüst es sich augentlich. 
lich in Atfler und $W$ eingeisl. Diese Auflöaungen besilzen ganz den Geschmack und Geruch des Bromals, sie reagiren weder sauer noch werden sie durch salpetersaures Silberoxyd gefält. Es verbindet sich selur Icicht mit Brom, und auch Phosphor und Schwefel werden davon aufgeiizat. Von Chlor und reuchender Salpetersäure wird es-zerselat, aber nicht von Chlorwaserstoffsäuro und Schrofelsäure. Als ein Kennzeichen seiner Reinheit kann das Verhalten au Schwefelstiure dienen: Eo darf nämlich damit zusammengebracht gar nicht rauchen; fandet dies statt, so hängt ihm noch schwerer Bromälher an. Wasserfreie Metalloxyde zinsacern keine Wirkung; wasserhaltige ahex zerbetzen es schon bei gelinder Wärne; man erhält Brommelall, Ameisenstiure und fliisoigen Bromkohlenstoff. Diese Zersetzung erfolgt ohne Farbenänderung und ohne Bildung einer gasförmigen Subsianz. L,citet man das Bromal über glühendes Eisen, so erhält man Bromeisen, Kohle und Kollenoxydgas. Werden die Dämpfe des Bromalg über erditzten wasserfreien Baryt oder Kalk geteilet, 80 werden diese beide glïhend, entwickelt sich Kollenoxydgas unter Erzeugtmg von Brommetall, welches mit Kohle vermischt iet.

Der Versuch mit glïhendem Eisen beweisst, dass das Bromal aus Brom, Kohlenstoff und Sauerstoff bestelen muss, und aleo wie das Chloral zusammengesetzt iut.

Wie beim Chlorkohlenstoff habe ich beim Bromal nur den Bromgehalt auf dieselbe Weise wie beim Bromkohlenstuff ausgemilteit. Derselbe stimul so sehr mit dern Culorgehalle des Chlorals überein, dass ich olne Bedenken auch die übrigen Substanzen wie im Chloral annehme.

1,sag Grm. Bromal gaben 2,273 Bromsilber. Diese entsprechen 0,956 Brom $=84,67$ p. C.

x,ogx Grm. Bromal gaben 2,201 Bronusilluer. Diese entsprechen 0,920 Brom $=84,32$ p. C. 
0,874 Orm. Bromal gaben 1,69\& Bromsiller. Diese entsprechen $0,7 \times 2$ Brom $=81,46$ p. C.

Nimmt man das Mittel dieser- Brombestinmungen, so erhält man 83,48 p. C. Brom. Komwen nun die iibrigen Substanzen eben so wie im ClJoral vor, to bestehen 100 Theile Bromal ans :

$$
\begin{gathered}
\text { 10,37 Rolilenstoff, } \\
6,15 \text { Sauerstoff, } \\
88,48 \text { Brom. } \\
\hline 100,00 \text { Bromal }
\end{gathered}
$$

Bcreclinet man diese Zahlen auf Atomen, 80 ergiebl sich:

\begin{tabular}{rlc}
9 Atome Kohlenstoff & $=54$ & berechnet \\
$4-$ Sauerstoff & $=32$ & 5,71 \\
$6-$ Brom & $=470,4$ & 84,54 \\
\hline 1 Atom Bromal & $=556,4$ & $100,00$.
\end{tabular}

Es frägt sich nun, auf welche Weise soll man die Zersetzung des Bromals durch Einwirkung von wässrigen Salzbasen in Brommetall, A meisensäure und Bromkohlenstoff erklä. ren? Liebig nimmt bekanntlich an, dass bei der Behandlung des Chlorals mit ätzenden Alkalien 5 Atome Chlor und 4 Atome Kohlenstoff Hüssigen Chlorkohlenstaff bilden, während 1 Atom Chlor mit 1 Atom Metall Chlormetall liefert, während das 1 Atom mit Metall verbundenem Sauerstoff mit. den noch vorhandeuen 5 At. Koll]ensioff und 4 At. Sauerstoff Kohlenoxydgas bildet, welches mit Wasser Ameisensäure hervorbringt.

Besieht der Bromkohlenstoff aus gleichen Atomen Brom and Kohlenstoff, so sucht man vergeblich nach einer passenden Formel, um die Zersetzung des Bromals in Ameisensäure und Brumkohlensloff zu erklären. Man könnte zwar annehmen, dass 5 A tome Brom snit 5 Atomen Kohlenstoff flïssigen Chlorkohlensloff biluen, und i Atome Kolbenstoff mil 4 Alomen 
Samerstoff Kohlenoxydgus, welches mit Wasser zu Ameisenstiure sich yereinigto, aber was wird in diesen Falle aus dem Sauerstoff des Melalioxyds, dessen Kadikal sich mit dem sechsItn Alom Chlor rereinig!? Bromsäire wird, wie ich mich auf das Bestimmnteste überzeugt, nicht gebildet. Lis ist also klar, dass entweder das Bromal eine von dem Chloral verschiedene Zusinmertselaung hat, welches jedoch nicht wahrsobeinlich ist, oder lass die Anuiyse des Aüssigen Bromkokstoffs unrichlig seyn muss. Pnilä̈lt nämlich der Bromkoh. leustoff nus eine hieine Menge unzersetztes Bromal, so mus uutiulich der Kohlenetuffgeliall au gross ausiallen. Es ist möglich, dass der zur Analyse benutzte Bromkohlenetoff noch etwas Bromal enthielt, und ich werde daker denselben nochmals untersuchen, und die Reguliate möglichst bald in diesen Annalen mittheilen. Ich babe auch antersucht, in welchem Vexhältniss das Brommelall zum ameisensauren Solze steht, aber ein Resuliat exhalten, welches ich um 80 weniger mittheile, da es weder für die eine, noch die andere Meinung enlocheidend ist.

\section{Broracallydrat.}

Wird das Bromal in einer Schale der Luft ausgeselzt, so erhäll man in kurzer Zeit eine Menge blendend weisser Krystalle, abne dass irgend cir Theil davon fliissig bleibt, die Bildung dieser Krysialle geht um so schneller vou statien, je malsr Oberthiche der luft dargehuten wird und je feuchter dicselbe ist. In ganz Irockner Luft, die man üler Chlorcalciun geleilet und ibe: Quecksilber aufgefengen bat, crfulgt sie nicht.

Setst man zu Broma! eine kieine Menge Wasser, so vermischt sicl: dasalle gleichformig damit, und man bemerkl anfangs keine Aloecheidung der kryslallisirlen Substanx. Lässt

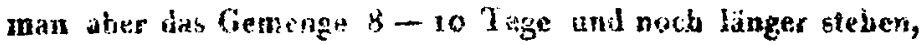
so crhält man gun gresse wasserklare und ausgezeichnet deut- 
liche Ksystalle von der Form des Kupfervilxiols. Ich besitze einen Krystall von der Lange eines Zolls. Diese Krystalle haben ganz der Geruch und Geschnack des Bronals, und sie sind Verbinilungen des Bromaln ntit Wasser. Mit Schwefessüure vermischt treters sie an diesellbe augenblinklich ihr Wasser ab, und man erhält wieder flüssiges Bromal. Die Krystalle sind wie das Bromal in Wasser vollständig löslish, und beim gelinden Verdunsten des Wuszers crkäll man wieder Krystulle. Bo die Bildung dieserRrystalle in Waseeraufnahme bestebi, so konnte ich leicht die Menge des letzlem finden, indem ich den Bromgelaalt der Krystalle bestimmte. Ich fand auf diese Weise, dass 1 Alom Bromal mit a Atom Wasser vereinigt ist. Werden diese Krystalle gepulvert und mit Wasser befeuchtet längere Zeit stehen gelassen, so erhält man kein zweites Hydrut des Bromals. Die Krystalle des Bromals schmelzen in gelinder Wirme, ja schon in der Wärme der Hand.

\section{Untersuchung der obersten Schicht.}

Wird die oberste Schicht auf dieselbe Weise wie die unterate Schicht behandelt, so crhält man ganz dieselben Resultałe; nur scheint in derselben mehr Bromsäure und Bromwasserstoffäure nebst Wasser enthalten zu seya, worin das geringere spec. Gew. zu suchen ist.

Da ich vermuthete, dass die Entstehung von schwerem Bromäther rom Vorhandenseyn kleiner Antheile Wasser im Alkohol herrïlsen könnte, so wurde eine neue Quantität $\Delta l$ kohol, von dessen $\mathbf{A b s o l u t i f u ̈ t}$ ich vollkommen überzeugt war, in demselben Apparate durch Brom zerlegt, aber mit der Abänderung, dass ich in die Flasche zuerst Brom brachte, es zum Kochen erhilzte und dann tropfenweise den Weingeist mit demselben in Berührung brachte; ich konnte bei dieser Abänderung vollkommen überzeugt seyn, dass die Zersetzung 
des Weingeists vollständig von stalten ging; wber alle Erschelnungen waren diesthen und zuletzt blieb wieder ein Gemenge von Bromal and schwerem Bromiather zuriick.

Aus den mitgethtilten Untersuchungen geht hervor, dass die Zersetzungsyrudukte des absululen Weingeists miltelst Brom folgende sind:

1) fesler Bromkohlensluff,

2) Bromkohlensauerstutt' (Bromal),

3) Ameisensäure,

4) Bromwasserstoffsäurc,

5) Bromwasserztoffnaphla

6) schwerer Bromäther,

7) Wasser,

8) eine weisse feste Substanz, dis nicht unteraucht werden konnte.

Der Weingeist wird also vollständig zerlegt, und zwar vereinigen sich dio Elemente im Momente ihres Freiwerdens 20 Verbindungen, die auf directe Weise nicht hervorgebrachit werden können.

Meine Inlersuchungen über die Zersetzung des Aethers durch Weingeisl, über den Bromkoh!enwassernioff und den schweren Bromälher u. s. w. werde ich nächstens mittheilen. 